\title{
DIAGNOSTIC CHALLENGES OF ORAL LEISHMANIASIS: UNUSUAL PRESENTATION OF TWO CASES
}

\author{
André Gebrim Matias*, Vinícius Gonçalves de Souza*, Eduardo Carreira Marcório**, Damilys \\ Joelly Souza Santos**, Tatiana Santos Araújo**, Aparecida de Lourdes Carvalho*, Ludimila Paula \\ Vaz Cardoso* and Carla Silva Siqueira Miranda*
}

\author{
*Academic Unit of Health Sciences, Federal University of Jataí, Jataí-GO, Brazil \\ ${ }^{* *}$ Municipal Health Secretariat of Jataí, Jataí-GO, Brazil
}

\begin{tabular}{|c|c|}
\hline ARTICLE INFO & ABSTRACT \\
\hline Article History: & Leishmaniasis is a non-contagious infectious disease and has a broad spectrum of clinical forms. \\
\hline $\begin{array}{l}\text { Received } 06^{\text {th }} \text { March, } 2021 \\
\text { Received in revised form } \\
11^{\text {th }} \text { April, } 2021 \\
\text { Accepted } 03^{\text {rd }} \text { May, } 2021 \\
\text { Published online } 26^{\text {th }} \text { June, } 2021 \\
\end{array}$ & $\begin{array}{l}\text { Isolated oral mucosal leishmaniasis is rare and challenges clinicians and pathologists. We report } \\
\text { two cases of oral leishmaniasis manifested as ulcerative growth on the palate and tongue nodule. } \\
\text { Histopathological analysis was performed and the final diagnosis was made with the visualization } \\
\text { of amastigote-like structures. Both cases had unusual clinical manifestations and many } \\
\text { differential diagnoses were considered, including syphilis, paracoccidioidomycosis and }\end{array}$ \\
\hline $\begin{array}{l}\text { Key Words: } \\
\text { Leishmaniasis, Oral manifestations, } \\
\text { Oral medicine, Pathology. }\end{array}$ & $\begin{array}{l}\text { mesenchymal or epithelial neoplasms. The broad spectrum of clinical manifestations and the } \\
\text { often-inconclusive findings in histopathology reinforce the importance of clinicopathological } \\
\text { correlation in diagnosis of leishmaniasis. }\end{array}$ \\
\hline
\end{tabular}

*Corresponding author: André Gebrim Matias

Copyright (C) 2021, André Gebrim Matias et al. This is an open access article distributed under the Creative Commons Attribution License, which permits unrestricted use, distribution, and reproduction in any medium, provided the original work is properly cited.

Citation: André Gebrim Matias, Vinícius Gonçalves de Souza, Eduardo Carreira Marcório, Damilys Joelly Souza Santos, Tatiana Santos Araújo, Aparecida de Lourdes Carvalho, Ludimila Paula Vaz Cardoso and Carla Silva Siqueira Miranda. 2021. "Diagnostic challenges of oral leishmaniasis: unusual presentation of two cases", International Journal of Development Research, 11, (06), 47890-47893.

\section{INTRODUCTION}

Leishmaniasis is a non-contagious infectious disease, caused by different species of protozoans of the genus Leishmania and which can cause skin and mucosal ulcers. In Brazil, the protozoan is transmitted by the bite of infected female sandflies of the genus Lutzomyia (Cruz et al., 2016). Changes in the organism that follow the presence of the parasite depend on the interaction between Leishmania's antigenicity and the host's immune response. Because of this parasite-host interaction, the clinical spectrum of leishmaniasis with cutaneous manifestations varies: the anergic pole (low or absent cellular immune response) includes the diffuse cutaneous form (DCL), and the hyperegic pole (high cellular immune response) includes the mucocutaneous form (MCL) and mucosal form (ML). The cutaneous form (CL), is inserted in the space between the two poles. Therefore, it is possible to distinct a wide variety in clinical and histopathological characteristics of the different forms: limited ulcers with scarce parasites, granuloma formation and lymphocyte infiltration (CL), infiltrated boards, non-ulcerated disseminated nodules and many parasites (DCL) and destructive lesions in palate and nasal septum and parasite scarcity/absence (MCL and ML) (Torres-Guerrero et al., 2017). Considering the great diversity of the clinical presentation of the leishmaniasis, it is essential to recognize atypical patterns of the disease and include them as a differential diagnosis in several cases. Thus, the present study aims to report two cases of unusual oral presentation of mucosal leishmaniasis and the challenge in diagnosis.admissions in reputed varsity. Now, here we enlist the proven steps to publish the research paper in a journal.

\section{CASE REPORTS}

Case 1: A 78-year-old woman with slow growing lesions on the palate and oropharynx. The patient also reported dysphagia and odynophagia and could not characterize the onset of the disease. The physical examination of the oral cavity showed that the patient was using an upper total prosthesis, which was dirty, smelly and in poor condition. She also said that the routine use of the prosthesis was causing constant pain. The lesions on the palate and oropharynx were characterized by sessile insertion, yellowish color, fibrous consistency and irregular surface and shape (Fig. 1A). Lymphadenopathy was absent and the patient denied injuries to her skin or mouth. An incisional biopsy was performed and the histopathologic sections stained with Hematoxylin and eosin (H\&E) showed proliferation of glandular tissue with intense chronic inflammatory infiltrate composed of lymphocytes and plasma cells, ductal ectasia with mucus inside and proliferation of myoepithelial 
components (Fig. 1B-C). Besides, Periodic Acid Schiff's (PAS) staining was negative for microorganisms and positive for mucus within the lumen of glandular proliferation (Fig. 1D). To rule out the hypothesis of lymphoid hyperplasia or malignant neoplasms, immunohistochemistry reactions were performed for CD3, CD4 and CD20, being positive only in normal inflammatory cells. The diagnosis was suggestive of a traumatic reaction and the management included the removal of the traumatic factor (prosthesis). After 40 days without using the prosthesis, the patient returned to the clinic reporting improvement in symptoms. On physical examination, the lesions had progressed well, with a reduction in the injured area, redness, swelling and pus (Fig. 1E). Even so, a new incisional biopsy was performed to evaluate the progression of the lesion. The analysis of the palate tissue showed a stratified squamous epithelium with hyperplasia, hydropic degeneration and exocytosis. In other areas, the lamina propria showed intense mixed inflammatory infiltrate, composed of lymphocytes, plasma cells and a few eosinophils. In some areas, there were macrophages containing structures similar to amastigote. In the oropharynx tissue, pseudoepitheliomatous hyperplasia and areas of ulceration were observed. The lamina propria presented intense mixed inflammatory infiltrate, with a predominance of chronicity. Through the infiltrate, several macrophages contained amastigote forms of parasite inside (Fig. 1F) were detected in Giemsa stain. The diagnosis was compatible with leishmaniasis and the patient was referred to multidisciplinary management. an incisional biopsy was performed and the histopathological analysis in H\&E showed epithelial hyperplasia, acanthosis and morphological changes in basal lane (Fig. 2D). An intense and diffuse inflammatory infiltrate in the lamina propria was also observed, with a predominance of macrophages and eosinophils that permeate the neural and muscle structures (Fig. 2E). In Giemsa stain, it was possible to see macrophages containing structures similar to amastigote inside (Fig. 2F). The diagnosis was compatible with leishmaniasis.

\section{DISCUSSION}

In South America, ML is usually caused by Leishmania braziliensis, an obligate intracellular protozoan. The disease is characterized by an exuberated cellular immune response with the release of proinflammatory cytokines such as IFN- $\gamma$ and Tumor Necrosis Factor Alpha (TNF- $\alpha$ ) (Torres-Guerrero et al., 2017). Clinically, the disease manifests itself as ulcerated lesions that appear mostly in the upper respiratory tract, nose and mouth that can appear years after the primary Leishmania infection (Azizi et al., 2005). Oral leishmaniasis as an isolated manifestation of ML is rare and the diagnosis is a challenge due to the wide variety of differential diagnosis, including infectious, granulomatous and neoplastic diseases (Almeida et al., 2016).

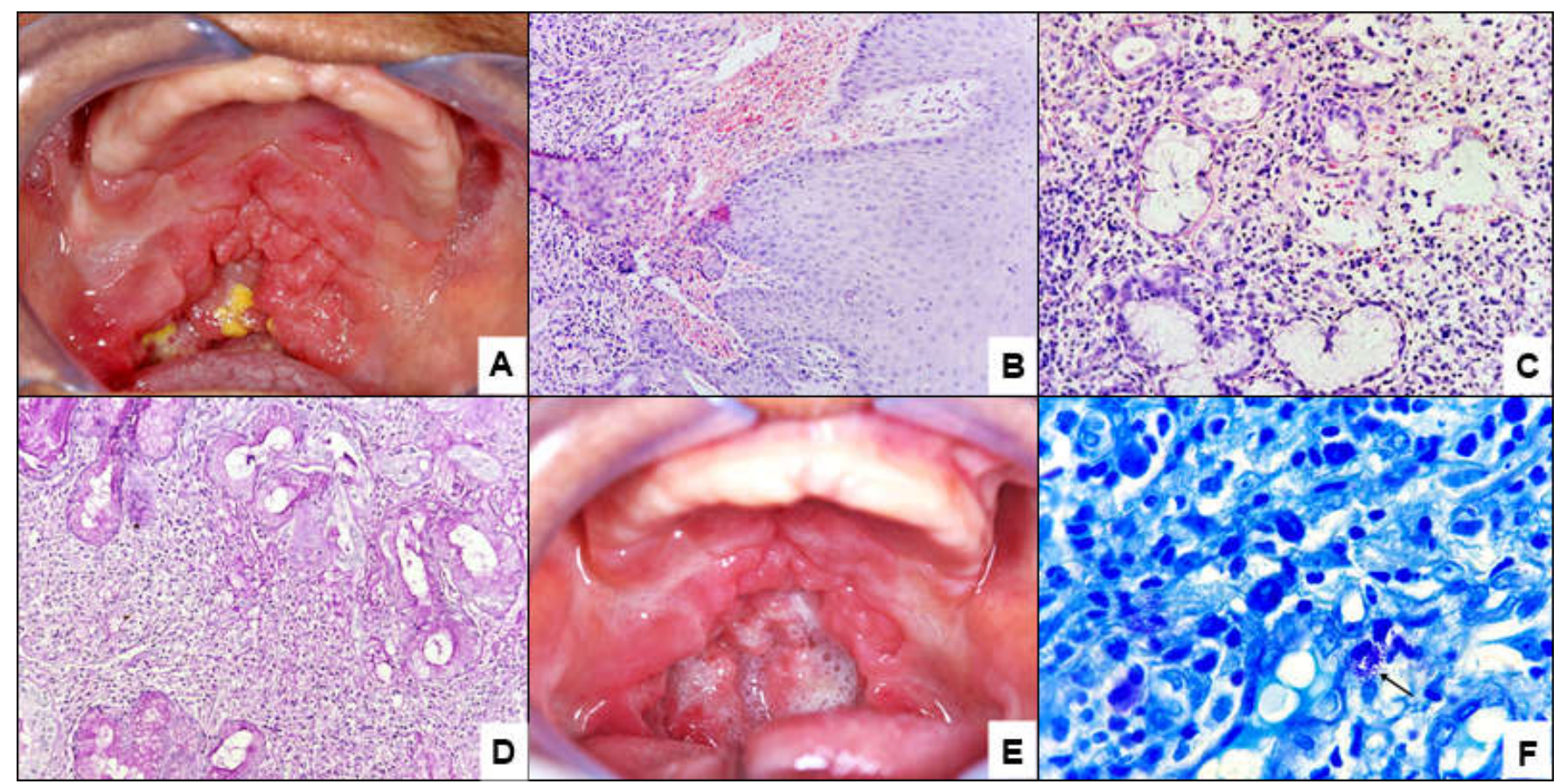

Figure 1. Oral leishmaniasis in a 78-year-old woman. In " $A$ " an ulcerative and irregular growth on the palate and oropharynx was observed, with yellowish color and fibrous consistency. In "B" and "C", stained with Hematoxylin and Eosin (H\&E) chronic inflammatory infiltrate composed of lymphocytes and plasma cells, ductal ectasia with mucus was observed and proliferation of myoepithelial components. PAS staining was positive for mucus within the lumen of glandular proliferation (D), $x 400$ magnification. After 40 days, the lesion progressed well after the removal of the prosthesis, with a reduction in the injured area, redness, swelling and pus (E). Black arrow indicate macrophage containing amastigote forms of parasite (F) in Giemsa stain. $x 1000$ magnification

Case 2: A 46-year-old man was attended in dental service with a 60days history of nodule on the right side of the tongue. The patient was smoker and alcoholic and denied the appearance of previous similar lesions in his skin and mouth. The physical examination of the oral cavity showed a mobile nodule on the right side of the tongue, measuring $0.7 \mathrm{~mm}$, painful to manipulation and increasing in size over time (Fig. 2A-B). For clinical investigation, a fine-needle aspiration (FNA) was performed, without complications and resistance of the patient at the time of the procedure. The cytological slides stained with Papanicolaou showed an abundance of purulent collection with large number of neutrophils and some macrophages, configuring a picture of acute inflammation (Fig. 2C). The possibility of an acute abscess has been suggested. In subsequent appointment,
In these situations, the clinical-pathological correlation is essential for the diagnosis, as highlighted in our cases. In the first case of this study, irregular growth of the palate was observed in a 78-year-old woman with no other comorbidities or history of cutaneous lesions. The literature review showed nine cases of ML with lesion on the palate. All the patients in the literature were men and the average age was 54.4 years (range, 28 to 94 years)(Almeida et al., 2016; Azizi et al., 2005; Costa et al., 2003; Cruz et al., 2016; Milián et al., 2002; Okumura et al., 2014; Palmeiro et al., 2007; Pellicioli et al., 2012). The large number of cases in adulthood and advanced age can be explained by the fact that lesions in the mucous membranes can appear after 20 years of primary Leishmania infection (TorresGuerrero et al., 2017). 


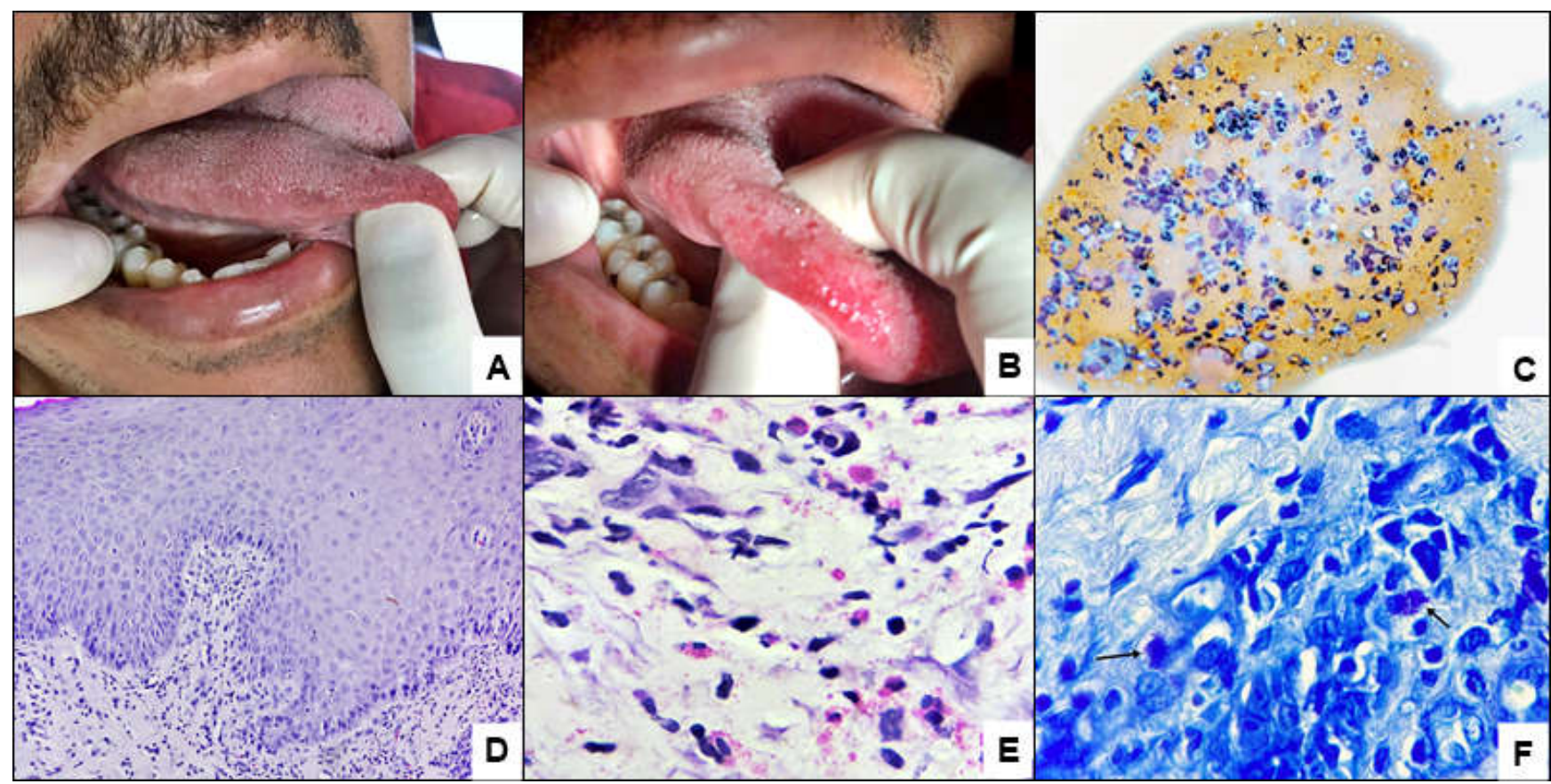

Figure 2. Oral leishmaniasis in a 46-year-old man. In "A" and "B", a mobile nodule can be seen on the right side of tongue. An abundant purulent collection with a large number of neutrophils and some macrophages was observed in the FNA material (C) stained with Papanicolaou. The histopathological analysis showed epithelial hyperplasia with morphological changes in baseline (D), intense diffuse inflammatory infiltrate rich in macrophages and eosinophils (E) stained with $H \& E$ and amastigote-like structures within macrophages indicated by black arrows (F) stained with Giemsa. C - E: x400 magnification. F: x1000 magnification

However, our patient had no related history of primary infection. The case presented in this study included painful ulcerative growth on the palate, with irregular size and shape. In the literature review, lesions on the palate were described as plaques (Almeida et al., 2016; Azizi et al., 2005), ulcerated nodules(Pellicioli et al., 2012), ulcerations (Costa et al., 2003; Milián et al., 2002), papillary lesions (Okumura et al., 2014) and granulomatous vegetations(Cruz et al., 2016; Palmeiro et al., 2007). Only five cases included isolated lesions of the palate (Almeida et al., 2016; Azizi et al., 2005; Milián et al., 2002; Palmeiro et al., 2007; Pellicioli et al., 2012), as in our case. It is important to note that the removal of traumatic factor (prosthesis) leads to an improvement in the clinical presentation, but ulcerative growth is still present. The main differential diagnosis cited in the literature included paracoccidioidomycosis, tuberculosis and squamous cell carcinoma (Palmeiro et al., 2007; Pellicioli et al., 2012). In our case, due to the clinical appearance, the hypothesis of lymphoma and lymphoid follicular hyperplasia related to trauma were also considered. In the second case, a 46-year-old man, smoker and alcoholic, presented with a mobile nodule on the tongue, without previous cutaneous lesions. The literature review showed nine cases of ML with tongue lesions (Baglieri\& Scuderi, 2012; Baily et al., 1994; Bueno-Filho et al., 2018; Diamantopoulos et al., 2006; Habibzadeh et al., 2005; Leitner et al., 2010; Mehlig et al., 2006; Mignogna et al., 2015; Pampín Franco et al., 2015). Tongue lesions appeared in eight men(Baglieri\& Scuderi, 2012; Baily et al., 1994; Bueno-Filho et al., 2018; Diamantopoulos et al., 2006; Habibzadeh et al., 2005; Leitner et al., 2010; Mehlig et al., 2006; Mignogna et al., 2015) and one woman(Pampín Franco et al., 2015), with an average age of 56.3 years (range, 39 to 71 years). In the reported case, the lesion on the tongue was noted as a mobile nodule, painful to handle and growing over time, but not ulcerated. The revised tongue lesions were described as ulcerations(Baglieri\& Scuderi, 2012; Baily et al., 1994; Bueno-Filho et al., 2018; Leitner et al., 2010), generalized edema (Diamantopoulos et al., 2006; Habibzadeh et al., 2005; Mehlig et al., 2006; Mignogna et al., 2015) and plaques(Pampín Franco et al., 2015). Therefore, we can observe that the clinical presentation of the tongue lesion in the reported case was atypical, since it presented as an intramucosal nodule, without ulceration, making the clinical suspicion of leishmaniasis difficult. The differential diagnosis of neurilemmoma, liposarcoma and Crohn's disease was also considered in this case.
The occurrence of isolated oral leishmaniasis in immunocompetent individual, such our patients, is uncommon(Habibzadeh et al., 2005) . However, patients often have several comorbidities that can influence disease progression. Regarding comorbidities, cases of oral leishmaniasis in the literature include high blood pressure(BuenoFilho et al., 2018; Pellicioli et al., 2012), human immunodeficiency virus (HIV) infection(Milián et al., 2002; Palmeiro et al., 2007), hepatitis $\mathrm{C}$ infection, smoking, alcoholism and drug abuse(Milián et al., 2002), lymphoma(Baily et al., 1994), ischemic heart disease(Baily et al., 1994), dyslipidemia(Bueno-Filho et al., 2018), kidney transplantation(Baglieri\& Scuderi, 2012), steatosis hepatitis(Leitner et al., 2010) and thymoma and thymectomy(Mehlig et al., 2006). Our patients were immunocompetent and only one had harmful habits, such as alcohol intake and smoking. Considering the diagnostic approach, in both case reports, the histopathological examination was essential for the diagnosis of leishmaniasis by viewing amastigote forms of parasite within macrophages on slides stained with H\&E or Giemsa. Finding amastigotes in the tissue in the ML is rare, and several of the reviewed cases have undergone multiple inconclusive biopsies. For the final diagnosis, in addition to the histopathological examination, other methods can be used, including molecular methods such as polymerase chain reaction (PCR) and immunological tests, such as immunofluorescence and enzyme-linked immunosorbent assay (ELISA)(Torres-Guerrero et al., 2017). The reported cases reinforce the importance of clinical suspicion of leishmaniasis in endemic areas. The broad spectrum of clinical manifestations and the often-inconclusive findings in histopathology reinforce the importance of the clinical-pathological correlation and the interaction between clinics and pathologists in the diagnosis of leishmaniasis.

\section{CONCLUSION}

The reported cases reinforce the importance of clinical suspicion of leishmaniasis in endemic areas. The broad spectrum of clinical manifestations and the often-inconclusive findings in histopathology reinforce the importance of the clinical-pathological correlation and the interaction between clinics and pathologists in the diagnosis of leishmaniasis 
Declarations: The authors declare no conflict of interest. All those who contributed to patient care participated in the writing of the article and are co-authors of the work. No funding was used in this work.

\section{REFERENCES}

Almeida, T. F. A., da Silveira, E. M., Dos Santos, C. R. R., León, J. E., \& Mesquita, A. T. M. (2016). Exclusive Primary Lesion of Oral Leishmaniasis with Immunohistochemical Diagnosis. Head and Neck Pathology, 10(4), 533-537. https://doi.org/10.1007/ s12105-016-0732-7

Azizi, T., Sadeghipour, A., Roohi, A., \& Nilipour, Y. (2005). A 33year-old male farmer with progressive gingival swelling and bleeding. Annals of Saudi Medicine, 25(3), 262,270-271. https://doi.org/10.5144/0256-4947.2005.262

Baglieri, F., \& Scuderi, G. (2012). A case of mucosal leishmaniasis of the tongue in a kidney transplant recipient. International Journal of Dermatology, 51(5), 597-600. https://doi.org/10.1111/j.1365-4632.2011.04981.x

Baily, G. G., Pitt, M. A., Curry, A., Haboubi, N. Y., Tuffin, J. R., \& Mandal, B. K. (1994). Leishmaniasis of the tongue treated with liposomal amphotericin B. The Journal of Infection, 28(3), 327331. https://doi.org/10.1016/s0163-4453(94)92193-8

Bueno-Filho, R., Vernal, S., Gomes, C. M., \& Roselino, A. M. (2018). Rare Tongue Compromising of Mucocutaneous Leishmaniasis by Leishmania Subgenus Viannia. Actas DermoSifiliograficas, 109(7), https://doi.org/10.1016/j.ad.2017.08.019

651.

Costa, J. W. J., Milner, D. A. J., \& Maguire, J. H. (2003). Mucocutaneous leishmaniasis in a US citizen. Oral Surgery, Oral Medicine, Oral Pathology, Oral Radiology, and Endodontics, 96(5), 573-577. https://doi.org/10.1016/s10792104(03)00299-3

Cruz, A. F., Resende, R. G., Albuquerque, D. R., de Lacerda, J. C. T., Leite, C. F., \& Ferreira Aguiar, M. C. (2016). Mucosal leishmaniasis in Brazilian patients: two case reports with similar clinical presentation and different approaches. Oral Surgery, Oral Medicine, Oral Pathology and Oral Radiology, 122(6), e199-e203. https://doi.org/10.1016/j.oooo.2016.02.017

Diamantopoulos, E. J., Andreadis, E. A., Tsourous, G. I., Petraki, C. D., \& Rontogianni, D. P. (2006). Persisting afebrile swelling of the lips and tongue: an unusual case of granulomatous glossitis. In The American journal of medicine (Vol. 119, Issue 2, pp. 182-183). https://doi.org/10.1016/j.amjmed.2005.06.065
Habibzadeh, F., Sajedianfard, J., \& Yadollahie, M. (2005). Isolated lingual leishmaniasis. Journal of Postgraduate Medicine, 51(3), 218-219. http://www.jpgmonline.com/article.asp?issn=00223859

Leitner, V., Weingast, J., Harmankaya, K., Walochnik, J., Pehamberger, H., Petzelbauer, P., Auer, H., \& Binder, M. (2010). Leishmaniasis in the tongue of an immunocompetent man. The American Journal of Tropical Medicine and Hygiene, 82(4), 597-599. https://doi.org/10.4269/ajtmh.2010.09-0698

Mehlig, A.-K., Arnold, A., Spicher, T., Koch, G., Hatz, C., \& Laifer, G. (2006). Late Relapse of Mucosal Leishmaniasis of the Tongue in a Patient After Thymectomy. Archives of Dermatology, 142(6), 781-798. https://doi.org/10.1001/ archderm.142.6.785

Mignogna, M. D., Celentano, A., Leuci, S., Cascone, M., Adamo, D., Ruoppo, E., \& Favia, G. (2015). Mucosal leishmaniasis with primary oral involvement: a case series and a review of the literature. Oral Diseases, 21(1), e70-8. https://doi.org/10.1111 /odi. 12268

Milián, M. A., Bagán, J. V, Jiménez, Y., Pérez, A., \& Scully, C. (2002). Oral leishmaniasis in a HIV-positive patient. Report of a case involving the palate. Oral Diseases, 8(1), 59-61. https://doi.org/10.1034/j.1601-0825.2002.1c666.x

Okumura, Y., Yamauchi, A., Nagano, I., Itoh, M., Hagiwara, K., Takahashi, K., Uezato, H., Maeda, M., \& Seishima, M. (2014). A case of mucocutaneous leishmaniasis diagnosed by serology. The Journal of Dermatology, 41(8), 739-742. https://doi.org/10.1111/1346-8138.12564

Palmeiro, M. R., Rosalino, C. M. V., Quintella, L. P., Morgado, F. N., da Costa Martins, A. C., Moreira, J., de Oliveira Schubach, A., \& Conceição-Silva, F. (2007). Gingival leishmaniasis in an HIV-negative patient. Oral Surgery, Oral Medicine, Oral Pathology, Oral Radiology, and Endodontics, 104(6), e12-6. https://doi.org/10.1016/j.tripleo.2007.07.008

Pampín Franco, A., Gamo Villegas, R., Caro-Gutiérrez, D., LópezEstebaranz, J. L., \& Pinedo, F. (2015). Mucosal leishmaniasis of the tongue caused by Leishmania infantum in an immunocompetent woman. In International journal of dermatology (Vol. 54, Issue 1, pp. e39-41). https://doi.org/10.1111/ijd.12567

Pellicioli, A. C. A., Martins, M. A. T., Sant'ana Filho, M., Rados, P. V, \& Martins, M. D. (2012). Leishmaniasis with oral mucosa involvement. Gerodontology, 29(2), e1168-71. https://doi.org/ 10.1111/j.1741-2358.2011.00512.x

Torres-Guerrero, E., Quintanilla-Cedillo, M. R., Ruiz-Esmenjaud, J., \& Arenas, R. (2017). Leishmaniasis: a review. F1000Research, 6, 750. https://doi.org/10.12688/f1000research.11120.1 